NISTIR 7153

\title{
The State of Container Security Standards
}

\author{
Shaw C. Feng \\ Simon P. Frechette
}


NISTIR 7153

\title{
The State of Container Security Standards
}

\author{
Shaw C. Feng \\ Simon P. Frechette \\ Manufacturing Engineering Laboratory
}

U.S. Department of Commerce

Carlos M. Gutierrez, Secretary

National Institute of Standards and Technology James M. Turner, Acting Director 


\section{Table of Contents}

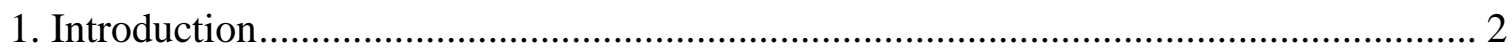

2. Container Security Standards Requirements ...................................................... 2

3. The Current State of Standards for Container Security ….......................................... 3

3.1 Standard Development Organizations .............................................................. 5

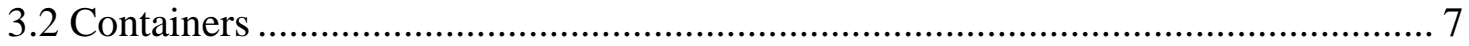

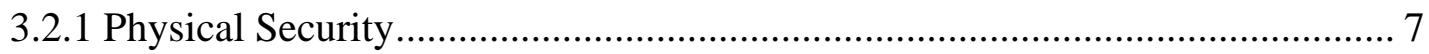

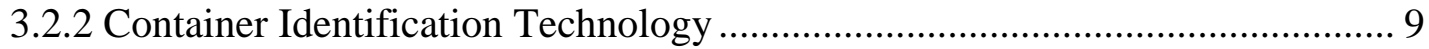

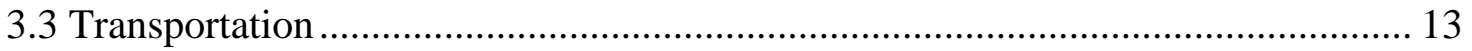

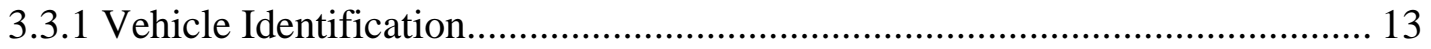

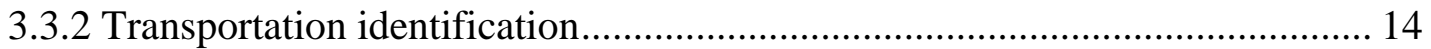

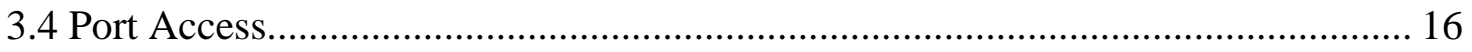

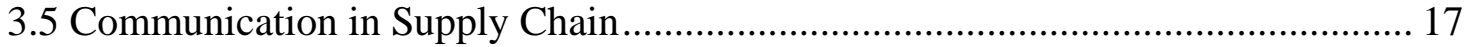

3.5.1 Medium and Long-Range Communication ............................................ 17

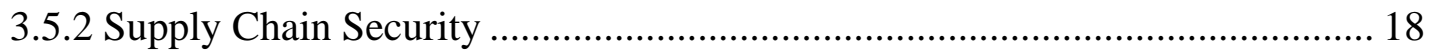

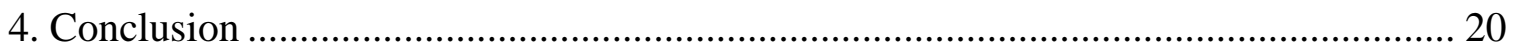

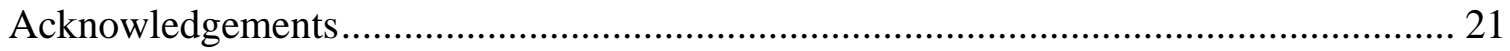

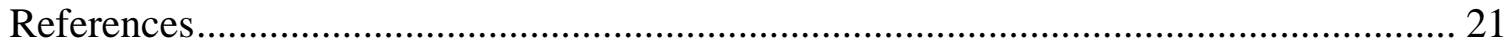




\section{Introduction}

About seven million containers arrive at U.S. ports every year. Almost 95\% of U.S.bound cargo moves by containers [3]. Currently, U.S. Customs officials use advanced technologies to identify and prescreen high-risk containers. The Department of Homeland Security has issued security guidelines for U.S. agencies, carriers, and importers in the Customs Trade Partnership Against Terrorism (C-TPAT). C-TPAT encourages importers and carriers to certify their cargo sources and assume responsibilities for cargo security [1].

The RAE report [3] stresses the importance of integrating sensor networks with supply chain databases and existing import/export processes to allow global traders to manage and monitor their maritime cargo in transit. Effective sensor technology and integrated information systems will be critical components of a comprehensive container inspection system. Data communication standards play a key role in direct data flow from sensors, electronic seals, and identification (ID) tags to location tracking and other systems used to provide overall shipping security.

Deployment of advanced container security technology is impeded by the lack of syntax and semantics standards for data exchange. This has led to proprietary solutions that work at isolated locations, but cannot be tied together for seamless data flow and data verification. Aside from the costs incurred by manually handling the same data numerous times and the errors that inevitably result, the lack of standards inhibits the deployment of truly intelligent systems that can leverage today's computer technology. Such technology promises the ability to track a container's history, pinpoint security breaches, recognize emerging patterns and anomalies, and automatically alert the downstream and upstream transit points.

This report focuses on the standards needed to integrate security device outputs and cargo processing information into supply chain information systems. It provides a survey of the state of the art in standards that address container security.

\section{Container Security Standards Requirements}

Both the Container Security Initiative (CSI) [1] and C-TPAT have raised new security requirements on cargo container standards. Physical integrity, communication between sensors, identification methodologies including Radio Frequency Identification (RFID), supply chain systems, and container shipping information repository systems are within the scope. The following information standard needs can be derived from CSI and CTPAT.

- Specification of physical/structural security

- Seal tampering detection and reporting

- Tracking, tracing, and condition reporting

- Unique identifying importer, carrier, and cargo source 
- Communication between electronic seals, RFID, and supply chain security databases

- Shipping route, including stops and departure/arrival dates

- Originator, transporters, recipient, cargo inspectors, and other container handlers and their security certifications

- Sequence of custodianship transfers, including transferring date, place, inspection, and other relevant information

\section{The Current State of Standards for Container Security}

All the currently available standards address the security of containers in transit. A container contains products that are ordered by clients and manufactured by a supplier. The container is transported by truck, trains, or ship. At each national boundary, the container has to be checked by customs. While in transit, the container is usually tracked by using satellite or land-based communication. Electronic door seals, radio frequency identification tags, supply chain tags, vehicle identification tags, and container ship identification devices are used to provide real-time container identification, location, and intrusion detection. As shown in Figure 1, containers to be shipped overseas are loaded and then transported to a customs check point at a sea port.

Standard Development Organizations (SDOs) are described in Section 3.1. Currently available port access security standards are described in Section 3.4. The cargo owner and shipper can track the container using land-based and satellite-based methods. Satellites provide real-time location and communication capabilities. Communication and air-interface standards are described in Section 3.5. At a sea port, the container is loaded to a container ship. The container goes through customs and port security check on land at the port of origin. At the destination, the container is loaded off from the ship to a delivery vehicle. The container could be loaded to different modes of transportation on land, such as truck, trailer, or train. It might also be temporarily off loaded for storage during intermodal transportation. Supply chain security standards including eBusiness standards and management system standards are described in Section 3.5.2.

Technical areas in container and vehicle security standards are shown in Figure 2. Those standards address both physical security and identification. Physical security includes electronic and mechanical door seals and structural integrity. Container identification (ID) has in two categories: individual container ID and supply chain ID. Details are described in Section 3.2. Also, details of vehicle-related security standards (tagging, registration, and intermodal transportation) are described in Section 3.3. 


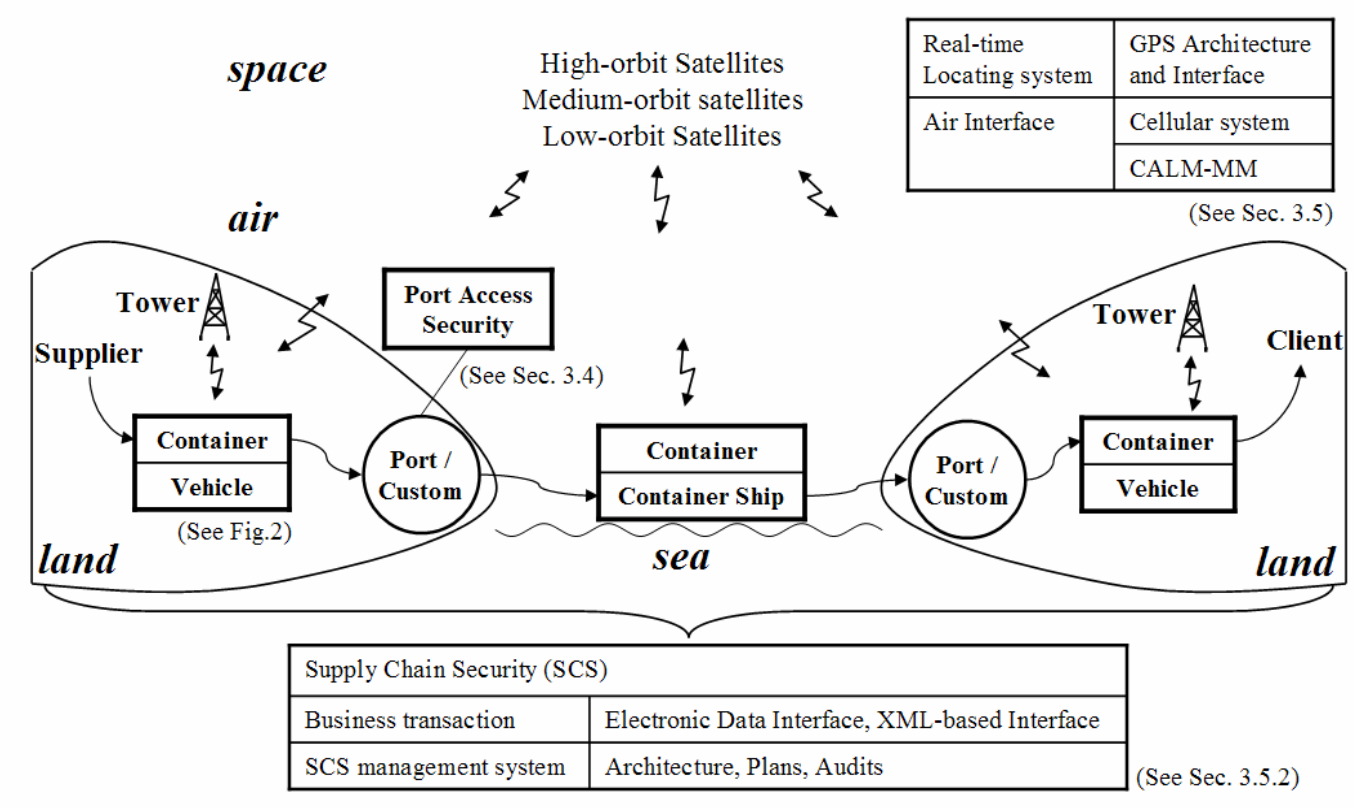

Figure 1 Container in a supply chain

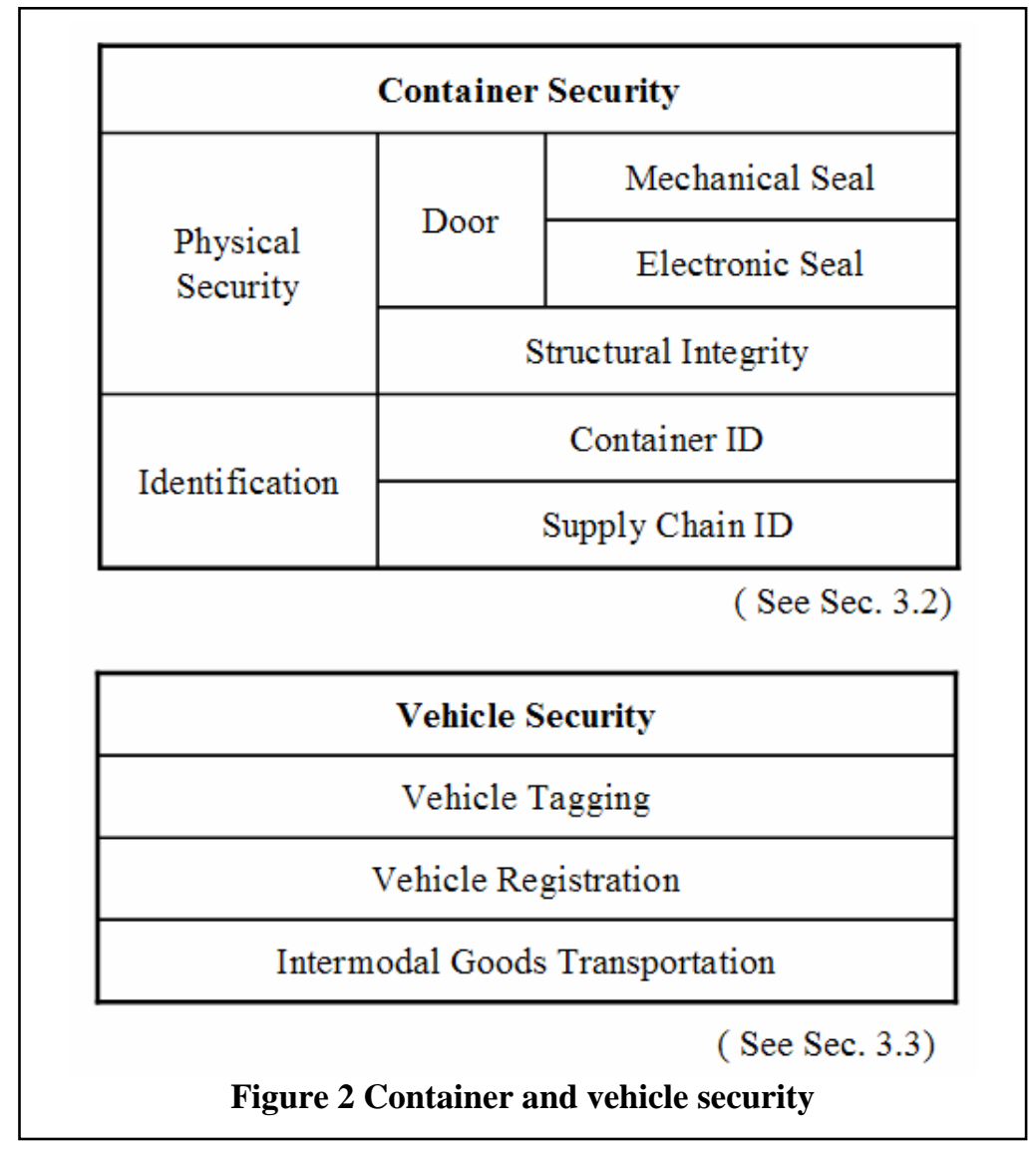




\subsection{Standard Development Organizations}

Most available standards were developed under the auspices of the International Organization for Standardization (ISO) [4]. The standards can be categorized by their functions as follows: container security, transportation security, port security, and supply chain communication security. The goal of standards development is to have a complete set of standards that are critical to the security of the supply chain, from origin to final destination. Many available ISO standards are listed in the ascending order of the standard number starting on [7] in References; a complete list of such standards is beyond the scope of this report. It is the authors' intent to keep updating this report as new standards become available.

There are generally six stages in the development of an ISO standard. The first stage is standard proposal. A new work item proposal (NP) is submitted for vote by members of the relevant Technical Committee (TC) or Subcommittee (SC) to determine the inclusion of the newly proposed work time. The second stage is standard preparation. A working group of technical experts, led by a project leader, prepare a Working Draft (WD) until the working group is satisfied that it has developed the best possible solution to solve the problem being addressed. The draft is thus forwarded to the parent committee for consensus building. The third stage is committee stage. The working draft is registered by the ISO Central Secretariat as a Committee Draft (CD) and distributed for comment. Successive CD revisions may be necessary until consensus is reached. Sometime, voting by qualified voting members takes place to reject the CD if consensus cannot be reached. Once consensus has been attained, the document is finalized for submission as a Draft International Standard (DIS). The fourth stage is enquiry. The DIS is circulated for all ISO members for voting and comment within a specified period. It may be approved for submission as a Final Draft International Standard (FDIS) or disapproved and returned to the committee for rework. The fifth stage is approval. The FDIS is circulated to all ISO member bodies for the final approval. The sixth stage is publication. Once the FDIS has been accepted by ISO, the final text is published as an International Standard (IS). Those draft standards that consensus is not attained can be published as a Technical Report (TR), Technical Specification (TS), or Publicly Available Specification (PAS), decided by the working group, SC, or TC to publish the useful information. They follow a less rigorous review and approval process than that of an IS.

An IS can be amended later. Amendment (Amd) is attached to the IS title. In addition to ISO, The International Electrotechnical Commission (IEC) [5] forms the Joint Technical Committee (JTC) with ISO to developed standards of common interests.

ISO committees that develop container security-related standards are as follows.

ISO/TC8 (Ships and Marine Technology) develops standards for shipbuilding, ship operations, sea-going ships, vessels for inland navigation, offshore structures, ship-toshore interfaces, and other marine structures required by the shipbuilding industry. Shipbuilding standards address design, construction, structural elements, outfitting parts, equipment, methods and technology, and marine environmental matters. 
ISO/TC 104 (Freight containers) develops standards of terminology, classification, dimensions, specifications, handling, test methods, and marking for freight containers that have an external volume of at least one cubic meter.

ISO/TC 122 (Packaging) develops standards in the field of packaging with regard to terminology and definitions, packaging dimensions, performance requirements, and tests.

ISO/TC 104/TC 122 Joint Working Group develops standards for supply chain applications of RFID for packages, means of packaging, and unit loads. These standards address technical areas of performance requirements and tests.

ISO/TC 154 (Processes, data elements and documents in commerce, industry and administration) develops standards for business transactions, administration processes, and global supply chain supporting data.

ISO/TC 204 (Intelligent transport systems) develops standards for information, communication, and control systems for urban and rural surface transportation, including traveler information, traffic management, public transport, commercial transport, emergency services, and commercial services. The standards address issues in both intermodal and multimodal transportation.

ISO/IEC JTC1/SC17 (Cards and personal identification) develops standards in the following technical areas: physical characteristics and test methods for ID cards, machine readable ID cards, integrated circuit card with or without contacts, registration management, optical memory cards and devices, motor vehicle driver licenses, and applications of biometrics to cards and personal identification.

JTC1/SC 31 (Automatic identification and data capture techniques) develops standards in the technical areas of data carrier, data structure, conformance tests, RFID for item management, and real time locating systems.

JTC1/SC 37 (Biometrics) develops standards in the technical areas of harmonized biometric vocabulary, biometric system interface, biometric data interchange formats, biometric functional architecture and profiles, and biometric software testing and reporting.

In addition to ISO and IEC, the World Customs Organization (WCO) is an independent intergovernmental organization that is to coordinate Customs administrations among countries. With 169 Member Governments, it is the only intergovernmental worldwide organization competent in Customs matters. WCO has the following primary functions: - harmonization and uniform application of agreed Customs systems and procedures governing the movement of commodities, people, and conveyances across Customs frontiers,

- Reinforcement of Members' compliance with their legislation and co-operation to combat Customs and other transnational offences, and 
- Promotion of communication and co-operation among Members and with other international organizations, and by fostering integrity, human resource development, transparency, improvements in the management and working methods of Customs administrations and the sharing of best practices.

WCO has developed a Framework of Standards to Secure and Facilitate Global Trade in 2005 [6].

The International Maritime Organization (IMO), formerly known as the InterGovernmental Maritime Consultative Organization (IMCO), was established in 1948 through the United Nations to coordinate international maritime safety and related practices. However, the IMO did not enter into full force until 1958. The organization promotes cooperation among governments and the shipping industry to improve maritime safety and to prevent marine pollution. IMO is governed by an Assembly of members and has other functions of marine safety and environmental protection.

The International Container Bureau is an organization with the goal to unite corporations or government units that handle, process, transport, and inspect freight containers. It has several publications and is responsible for the Bureau International des Conteneurs (BIC) codes, which constitute the standard ISO 6346 [10] coding system for every container owner and container operating company. The Bureau is located in Paris, France.

The World Shipping Council is a trade association representing more than forty liner shipping companies serving America's international trade. Located in Washington, D.C., members include container lines and carriers. In addition to ocean transportation, members provide a wide range of intermodal and logistics services to American importers and exporters. Issues addressed by the Council include maritime security, regulatory policy, safety, harbor dredging, and upgrading the shipping infrastructure.

The developed container security-related standards are described in the following sections.

\subsection{Containers}

Container standards are categorized into physical security and container identification technology. A vocabulary for freight containers is specified in ISO 830 [7].

\subsubsection{Physical Security}

Physical security includes door security, structural integrity, and seals. Standards for door security are in ISO 1496 Part 1 . Standards for container structural integrity and tests are specified in ISO 15070 and Amendment 1. Recently, standards for container seals have been developed. They include ISO 17712 for bolt locks and the ISO 18185 series for electronic seals [2]. 


\begin{tabular}{|c|c|c|c|c|}
\hline \multicolumn{3}{|c|}{ Cargo Container Physical Security } & SDO & Status \\
\hline \multirow{5}{*}{ Door } & \multicolumn{2}{|c|}{$\begin{array}{l}\text { ISO } 1496 \text { Series } 1 \text { freight containers - Specification and } \\
\text { testing - Part 1: General cargo containers for general } \\
\text { purposes [8] }\end{array}$} & ISO/TC104 & IS \\
\hline & \multicolumn{2}{|c|}{ ISO 1496 Part 1/Amd 5:2006 Door end security [9] } & ISO/TC104 & IS \\
\hline & \multicolumn{2}{|c|}{$\begin{array}{l}\text { ISO } 15070 \text { Series } 1 \text { freight containers - Rationale for } \\
\text { structural test criteria [32] }\end{array}$} & ISO/TC104 & TR \\
\hline & \multicolumn{2}{|c|}{ ISO 15070/Amd 1 Guidance on structural integrity [33] } & ISO/TC104 & TR \\
\hline & \multicolumn{2}{|c|}{ ISO 15070/Amd 2 Design Consideration [34] } & ISO/TC104 & $\mathrm{CD}$ \\
\hline \multirow{8}{*}{ Seal } & Mechanical & $\begin{array}{l}\text { ISO } 17712 \text { Freight containers - Mechanical } \\
\text { Seals [57] }\end{array}$ & ISO/TC104 & PAS \\
\hline & \multirow[t]{7}{*}{ Electronic } & $\begin{array}{l}\text { ISO } 18185 \text { Freight containers - Electronic } \\
\text { seals - Part 1: Communication protocol [71] }\end{array}$ & ISO/TC104 & FDIS \\
\hline & & $\begin{array}{l}\text { ISO } 18185 \text { Freight containers - Electronic } \\
\text { seals - Part 2: Application requirements } \\
\text { [72] }\end{array}$ & ISO/TC104 & FDIS \\
\hline & & $\begin{array}{l}\text { ISO } 18185 \text { Freight containers - Electronic } \\
\text { seals - Part 3: Environment characteristics } \\
\text { [73] }\end{array}$ & ISO/TC104 & IS \\
\hline & & $\begin{array}{l}\text { ISO } 18185 \text { Freight containers - Electronic } \\
\text { seals - Part 4: Data protection [74] }\end{array}$ & ISO/TC104 & FDIS \\
\hline & & $\begin{array}{l}\text { ISO } 18185 \text { Freight containers - Electronic } \\
\text { seals - Part 5: Sensor interface [75] }\end{array}$ & ISO/TC104 & FDIS \\
\hline & & $\begin{array}{l}\text { ISO } 18185 \text { Freight containers - Electronic } \\
\text { seals - Part } 6 \text { Message sets for transfer } \\
\text { between seal reader and host computer [76] }\end{array}$ & ISO/TC104 & CD \\
\hline & & $\begin{array}{l}\text { ISO } 18185 \text { Freight containers - Electronic } \\
\text { seals - Part } 7 \text { Physical layer [77] }\end{array}$ & ISO/TC104 & DIS \\
\hline
\end{tabular}




\subsubsection{Container Identification Technology}

Radio Frequency Identification (RFID) technology is available for container identification. There are standards for air interfaces, implementation guidelines, system management, data content, data protocol, and performance tests for RFID used on containers.

\begin{tabular}{|c|c|c|c|}
\hline \multicolumn{2}{|r|}{ RFID } & SDO & Status \\
\hline \multirow{8}{*}{ Air interface } & $\begin{array}{l}\text { ISO/IEC Information Technology - Radio } \\
\text { frequency identification for item } \\
\text { management - Part 1: Reference architecture } \\
\text { and definition of parameters to be } \\
\text { standardize [58] }\end{array}$ & $\begin{array}{l}\text { ISO/IEC } \\
\text { JTC1/SC31 }\end{array}$ & IS \\
\hline & $\begin{array}{l}\text { ISO/IEC } 18000 \text { Information Technology - } \\
\text { Radio frequency identification for item } \\
\text { management - Part 2: Parameters for air } \\
\text { interface communication below } 135 \mathrm{kHz} \text { [59] }\end{array}$ & $\begin{array}{l}\text { ISO/IEC } \\
\text { JTC1/SC31 }\end{array}$ & IS \\
\hline & $\begin{array}{l}\text { ISO/IEC } 18000 \text { Information Technology - } \\
\text { Radio frequency identification for item } \\
\text { management - Part 3: Parameters for air } \\
\text { interface communication below 13,56 MHz } \\
\text { [60] }\end{array}$ & $\begin{array}{l}\text { ISO/IEC } \\
\text { JTC1/SC31 }\end{array}$ & IS \\
\hline & $\begin{array}{l}\text { ISO/IEC } 18000 \text { Information Technology - } \\
\text { Radio frequency identification for item } \\
\text { management - Part 4: Parameters for air } \\
\text { interface communication below } 2,45 \mathrm{GHz} \\
\text { [61] }\end{array}$ & $\begin{array}{l}\text { ISO/IEC } \\
\text { JTC1/SC31 }\end{array}$ & IS \\
\hline & $\begin{array}{l}\text { ISO/IEC } 18000 \text { Information Technology - } \\
\text { Radio frequency identification for item } \\
\text { management - Part 6: Parameters for air } \\
\text { interface communication at } 860 \mathrm{MHz} \text { to } 960 \\
\mathrm{MHz} \text { [62] }\end{array}$ & $\begin{array}{l}\text { ISO/IEC } \\
\text { JTC1/SC31 }\end{array}$ & IS \\
\hline & $\begin{array}{l}\text { ISO/IEC } 18000 \text { Part 6/Amd } 1 \text { Extension with } \\
\text { Type C and update of Types A and B [63] }\end{array}$ & $\begin{array}{l}\text { ISO/IEC } \\
\text { JTC1/SC31 }\end{array}$ & IS \\
\hline & $\begin{array}{l}\text { ISO/IEC FDIS } 18000 \text { Information } \\
\text { technology - Radio frequency identification } \\
\text { for item management - Part 7: Parameters for } \\
\text { active air interface communications at } 433 \\
\text { MHz [64] }\end{array}$ & $\begin{array}{l}\text { ISO/IEC } \\
\text { JTC1/SC31 }\end{array}$ & FDIS \\
\hline & $\begin{array}{l}\text { ISO } 24753 \text { Automatic Identification and Data } \\
\text { Capture Techniques - Radio Frequency }\end{array}$ & $\begin{array}{l}\text { ISO/IEC } \\
\text { JTC1/SC31 }\end{array}$ & NP \\
\hline
\end{tabular}




\begin{tabular}{|c|c|c|c|}
\hline \multicolumn{2}{|r|}{ RFID } & \multirow[t]{2}{*}{ SDO } & \multirow[t]{2}{*}{ Status } \\
\hline & $\begin{array}{l}\text { Identification (RFID) for Item Management - } \\
\text { Air Interface for Battery Assist and Sensor } \\
\text { Function [116] }\end{array}$ & & \\
\hline \multirow{3}{*}{$\begin{array}{l}\text { Implementation } \\
\text { guideline }\end{array}$} & $\begin{array}{l}\text { ISO/IEC } 24729 \text { Information technology - } \\
\text { Radio frequency identification for item } \\
\text { management - Implementation guidelines - } \\
\text { Part 1: RFID-enabled labels [109] }\end{array}$ & $\begin{array}{l}\text { ISO/IEC } \\
\text { JTC1/SC31 }\end{array}$ & $\begin{array}{l}\text { TR, } \\
\text { CD }\end{array}$ \\
\hline & $\begin{array}{l}\text { ISO/IEC } 24729 \text { Information technology - } \\
\text { Radio frequency identification for item } \\
\text { management - Implementation guidelines - } \\
\text { Part 2: Recyclability of RF tags [110] }\end{array}$ & $\begin{array}{l}\text { ISO/IEC } \\
\text { JTC1/SC31 }\end{array}$ & $\begin{array}{l}\text { TR, } \\
\text { CD }\end{array}$ \\
\hline & $\begin{array}{l}\text { ISO/IEC } 24729 \text { Information technology - } \\
\text { Radio frequency identification for item } \\
\text { management - Implementation guidelines - } \\
\text { Part 3: RFID interrogator/antenna installation } \\
\text { [111] }\end{array}$ & $\begin{array}{l}\text { ISO/IEC } \\
\text { JTC1/SC31 }\end{array}$ & $\begin{array}{l}\text { TR, } \\
\text { NP }\end{array}$ \\
\hline $\begin{array}{c}\text { System } \\
\text { management }\end{array}$ & $\begin{array}{l}\text { ISO/IEC } 24791 \text { Information technology - } \\
\text { Automatic Identification and Data Capture } \\
\text { Techniques - Radio-Frequency Identification } \\
\text { (RFID) for Item Management - System } \\
\text { Management Protocol [117] }\end{array}$ & $\begin{array}{l}\text { ISO/IEC } \\
\text { JTC1/SC31 }\end{array}$ & NP \\
\hline \multirow[t]{6}{*}{ Data content } & $\begin{array}{l}\text { ISO/IEC } 15418 \text { Information technology - } \\
\text { EAN/UCC application identifiers and fact } \\
\text { data identifiers and maintenance [35] }\end{array}$ & $\begin{array}{l}\text { ISO/IEC } \\
\text { JTC1/SC31 }\end{array}$ & IS \\
\hline & $\begin{array}{l}\text { ISO/IEC } 15424 \text { Information technology - } \\
\text { Automatic identification and data capture } \\
\text { techniques - Data carrier identifiers [36] }\end{array}$ & $\begin{array}{l}\text { ISO/IEC } \\
\text { JTC1/SC31 }\end{array}$ & IS \\
\hline & $\begin{array}{l}\text { ISO/IEC } 15434 \text { Information technology - } \\
\text { Automatic identification and data capture } \\
\text { techniques - Syntax for high-capacity ADC } \\
\text { media [37] }\end{array}$ & $\begin{array}{l}\text { ISO/IEC } \\
\text { JTC1/SC31 }\end{array}$ & IS \\
\hline & $\begin{array}{l}\text { ISO/IEC } 15459 \text { Information technology - } \\
\text { Unique identifiers - Part 1: Unique identifiers } \\
\text { for transport units [38] }\end{array}$ & $\begin{array}{l}\text { ISO/IEC } \\
\text { JTC1/SC31 }\end{array}$ & IS \\
\hline & $\begin{array}{l}\text { ISO/IEC } 15459 \text { Information technology - } \\
\text { Unique identifiers - Part 2: Registration } \\
\text { procedures [39] }\end{array}$ & $\begin{array}{l}\text { ISO/IEC } \\
\text { JTC1/SC31 }\end{array}$ & IS \\
\hline & $\begin{array}{l}\text { ISO/IEC } 15459 \text { Information technology - } \\
\text { Unique identifiers - Part 3: Common rules for } \\
\text { unique identifiers [40] }\end{array}$ & $\begin{array}{l}\text { ISO/IEC } \\
\text { JTC1/SC31 }\end{array}$ & IS \\
\hline
\end{tabular}




\begin{tabular}{|c|c|c|c|}
\hline \multicolumn{2}{|r|}{ RFID } & \multirow{2}{*}{$\begin{array}{c}\text { SDO } \\
\text { ISO/IEC } \\
\text { JTC1/SC31 }\end{array}$} & \multirow{2}{*}{\begin{tabular}{|c|} 
Status \\
IS
\end{tabular}} \\
\hline & $\begin{array}{l}\text { ISO/IEC } 15459 \text { Information technology - } \\
\text { Unique identifiers - Part 4: Unique identifiers } \\
\text { for supply chain management [41] }\end{array}$ & & \\
\hline & $\begin{array}{l}\text { ISO/IEC } 15459 \text { Information technology - } \\
\text { Unique identification of transport units - Part } \\
\text { 5: Unique identification of returnable } \\
\text { transport items (RTIs) [42] }\end{array}$ & $\begin{array}{c}\text { ISO/IEC } \\
\text { JTC1/SC31 }\end{array}$ & FCD \\
\hline & $\begin{array}{l}\text { ISO/IEC } 15459 \text { Information technology - } \\
\text { Unique identification of transport units - Part } \\
\text { 6: Unique identification for product } \\
\text { groupings in material lifecycle management } \\
\text { [43] }\end{array}$ & $\begin{array}{c}\text { ISO/IEC } \\
\text { JTC1/SC31 }\end{array}$ & FCD \\
\hline \multirow{3}{*}{ Data protocol } & $\begin{array}{l}\text { ISO/IEC } 15961 \text { Information technology - } \\
\text { Radio frequency identification (RFID) for } \\
\text { item management - Data protocol: } \\
\text { application [44] }\end{array}$ & $\begin{array}{c}\text { ISO/IEC } \\
\text { JTC1/SC31 }\end{array}$ & IS \\
\hline & $\begin{array}{l}\text { ISO/IEC } 15962 \text { Information technology - } \\
\text { Radio frequency identification (RFID) for } \\
\text { item management - data protocol - encoding } \\
\text { rules and logical memory functions [45] }\end{array}$ & $\begin{array}{c}\text { ISO/IEC } \\
\text { JTC1/SC31 }\end{array}$ & IS \\
\hline & $\begin{array}{l}\text { ISO/IEC } 15963 \text { Information technology - } \\
\text { Radio frequency identification for item } \\
\text { management - unique identification for RF } \\
\text { tags [46] }\end{array}$ & $\begin{array}{c}\text { ISO/IEC } \\
\text { JTC1/SC31 }\end{array}$ & IS \\
\hline \multirow[t]{4}{*}{$\begin{array}{l}\text { Performance } \\
\text { tests }\end{array}$} & $\begin{array}{l}\text { ISO/IEC } 18046 \text { Information technology - } \\
\text { Automatic identification and data capture } \\
\text { techniques - Radio frequency identification } \\
\text { device performance test methods [65] }\end{array}$ & $\begin{array}{c}\text { ISO/IEC } \\
\text { JTC1/SC31 }\end{array}$ & IS \\
\hline & $\begin{array}{l}\text { ISO/IEC } 18047 \text { Information technology - } \\
\text { Radio frequency identification device } \\
\text { conformance test methods - Part 2: Test } \\
\text { methods for air interface communications } \\
\text { below } 135 \mathrm{kHz} \text { [66] }\end{array}$ & $\begin{array}{c}\text { ISO/IEC } \\
\text { JTC1/SC31 }\end{array}$ & TR \\
\hline & $\begin{array}{l}\text { ISO/IEC TR } 18047 \text { Information technology - } \\
\text { Radio frequency identification device } \\
\text { conformance test methods - Part 3: Test } \\
\text { methods for air interface communications at } \\
\text { 13,56 MHz [67] }\end{array}$ & $\begin{array}{c}\text { ISO/IEC } \\
\text { JTC1/SC31 }\end{array}$ & TR \\
\hline & ISO/IEC 18047 Information technology - & ISO/IEC & $\mathrm{TR}$ \\
\hline
\end{tabular}




\begin{tabular}{|l|l|c|c|}
\hline \multicolumn{1}{|c|}{ RFID } & SDO & Status \\
\hline & $\begin{array}{l}\text { Radio frequency identification device } \\
\text { conformance test methods - Part 4: Test } \\
\text { methods for air interface communications at } \\
\text { 2,45 GHz [68] }\end{array}$ & JTC1/SC31 & \\
\cline { 2 - 4 } & $\begin{array}{l}\text { ISO/IEC TR 18047 Information technology - } \\
\text { Radio frequency identification device } \\
\text { conformance test methods - Part 6: Test } \\
\text { methods for air interface communications at } \\
\text { 860 MHz to 960 MHz [69] }\end{array}$ & JTC1/SC31 & TR \\
\cline { 2 - 4 } & $\begin{array}{l}\text { ISO/IEC TR 18047 Information technology - } \\
\text { Radio frequency identification device } \\
\text { conformance test methods - Part 7: Test } \\
\text { methods for active air interface } \\
\text { communications at 433 MHz [70] }\end{array}$ & ISO/IEC & TR \\
\hline
\end{tabular}

An RFID tag can be attached to a container to identify it as a unit, and multiple RFID tags can be attached to individual packages to identify the goods in the container for monitoring, tracing, and tracking.

\begin{tabular}{|c|l|c|c|}
\hline \multicolumn{2}{|c|}{ Container Identification in a Supply Chain } & SDO & Status \\
\hline \multirow{5}{*}{ Container ID } & $\begin{array}{l}\text { ISO 6346 Freight container - Coding, } \\
\text { Identification and Marking [10] }\end{array}$ & ISO/TC104 & IS \\
\cline { 2 - 4 } & $\begin{array}{l}\text { ISO 10374 Freight container - RF } \\
\text { Automatic Identification [21] }\end{array}$ & $\begin{array}{c}\text { ISO } \\
\text { JTC104/TC122 }\end{array}$ & CD \\
\hline & $\begin{array}{l}\text { ISO 17363 Supply chain application of } \\
\text { RFID - Freight Containers [51] }\end{array}$ & ISO TC122 & DIS \\
\cline { 2 - 4 } Supply chain ID & $\begin{array}{l}\text { ISO 17364 Supply chain applications of } \\
\text { RFID - Returnable transport items [52] }\end{array}$ & ISO TC122 & DIS \\
\cline { 2 - 4 } & $\begin{array}{l}\text { ISO 17365 Supply chain applications of } \\
\text { RFID - Transport units [53] }\end{array}$ & ISO TC122 & DIS \\
\cline { 2 - 4 } & $\begin{array}{l}\text { ISO/DIS 17367 Supply chain applications of } \\
\text { of RFID - Product tagging [55] }\end{array}$ & ISO TC122 & DIS \\
\cline { 2 - 4 } & $\begin{array}{l}\text { ISO/FDIS 17687 Transport Information } \\
\text { Systems - General Fleet Management and } \\
\text { Commercial Freight Operations - Data } \\
\text { dictionary and message sets for electronic } \\
\text { identification and monitoring of hazardous }\end{array}$ & ISO TC122 & DIS \\
\hline
\end{tabular}




\begin{tabular}{|l|l|l|l|}
\hline \multicolumn{2}{|c|}{ Container Identification in a Supply Chain } & SDO & Status \\
\hline & materials/dangerous goods [56] & & \\
\hline
\end{tabular}

Sensors can be placed in a container to detect abnormal vibrations and temperature changes. An electronic seal is a type of sensor that detects and reports tampering.

\subsection{Transportation}

In transportation, standards for automatic vehicle identification and personnel identification are available.

\subsubsection{Vehicle Identification}

Radio Frequency (RF) identification-related standards are applicable for land-based container carriers, such as trucks and trains. Applications include tractor tagging, chassis tagging, vehicle registration, and intermodal goods transport.

\begin{tabular}{|c|l|c|c|}
\hline \multicolumn{1}{|c|}{ Vehicle Identification } & SDO & Status \\
\hline \hline \multirow{5}{*}{$\begin{array}{c}\text { Vehicle } \\
\text { tagging }\end{array}$} & $\begin{array}{l}\text { ISO 14814 Road transport and traffic telematics - } \\
\text { Automatic vehicle and equipment identification - } \\
\text { Reference architecture and terminology [29] }\end{array}$ & $\begin{array}{l}\text { ISO/TC204 } \\
\text { Automatic vehicle and equipment identification - } \\
\text { System specifications [30] }\end{array}$ & ISO/TC204 \\
\cline { 2 - 4 } & $\begin{array}{l}\text { ISO 14816 Road transport and traffic telematics - } \\
\text { Automatic vehicle and equipment identification - } \\
\text { Numbering and data structure [31] }\end{array}$ & ISO/TC204 & TS \\
\hline \multirow{5}{*}{ Registration } & $\begin{array}{l}\text { ISO 24531 Intelligent transport systems - System } \\
\text { architecture, taxonomy and terminology - Using } \\
\text { XML in ITS standards, data registries and data } \\
\text { dictionaries [95] }\end{array}$ & ISO/TC204 & FDIS \\
\cline { 2 - 4 } & $\begin{array}{l}\text { ISO/CD 24533 Transport information and control } \\
\text { systems - Data dictionary and message set to } \\
\text { facilitate the movement of freight and its } \\
\text { intermodal transfer - Road transport information } \\
\text { exchanges [96] }\end{array}$ & ISO/TC204 & CD \\
\cline { 2 - 4 } & $\begin{array}{l}\text { ISO 24534 Automatic vehicle and equipment } \\
\text { identification - Electronic Registration } \\
\text { Identification (ERI) for vehicles - Part 1: } \\
\text { Architecture [97] }\end{array}$ & ISO/TC204 & TS, \\
CD & \\
\hline
\end{tabular}




\begin{tabular}{|c|c|c|c|}
\hline \multicolumn{2}{|r|}{ Vehicle Identification } & SDO & Status \\
\hline & $\begin{array}{l}\text { ISO/CD TS } 24534 \text { Automatic vehicle and } \\
\text { equipment identification - Electronic Registration } \\
\text { Identification (ERI) for vehicles - Part 2: } \\
\text { Operational requirements [98] }\end{array}$ & ISO/TC204 & $\begin{array}{l}\text { TS, } \\
\text { CD }\end{array}$ \\
\hline & $\begin{array}{l}\text { ISO/CD TS } 24534 \text { Automatic vehicle and } \\
\text { equipment identification - Electronic Registration } \\
\text { Identification (ERI) for vehicles - Part 3: Vehicle } \\
\text { data [98] }\end{array}$ & ISO/TC204 & $\begin{array}{l}\text { TS, } \\
\text { CD }\end{array}$ \\
\hline & $\begin{array}{l}\text { ISO/CD TS } 24534 \text { Automatic vehicle and } \\
\text { equipment identification - Electronic Registration } \\
\text { Identification (ERI) for vehicles - Part 4: Secure } \\
\text { communications using asymmetrical techniques } \\
\text { [100] }\end{array}$ & ISO/TC204 & $\begin{array}{l}\text { TS, } \\
\text { CD }\end{array}$ \\
\hline & $\begin{array}{l}\text { ISO } 24535 \text { Intelligent transport systems - } \\
\text { Automatic Vehicle Identification - Basic } \\
\text { electronic registration identification [101] }\end{array}$ & ISO/TC204 & $\begin{array}{l}\text { TS, } \\
\text { DIS }\end{array}$ \\
\hline \multirow{4}{*}{$\begin{array}{l}\text { Intermodal } \\
\text { good } \\
\text { transport }\end{array}$} & $\begin{array}{l}\text { ISO 17261, Intelligent transport systems - } \\
\text { Automatic vehicle and equipment identification - } \\
\text { Intermodal goods transport architecture and } \\
\text { terminology [47] }\end{array}$ & ISO/TC204 & TS \\
\hline & $\begin{array}{l}\text { ISO/TS } 17262 \text { Intelligent transport systems - } \\
\text { Automatic vehicle and equipment identification - } \\
\text { Intermodal goods transport - Numbering and data } \\
\text { structures [48] }\end{array}$ & ISO/TC204 & TS \\
\hline & $\begin{array}{l}\text { ISO/TS } 17263 \text { Intelligent transport systems - } \\
\text { Automatic vehicle and equipment identification - } \\
\text { Intermodal goods transport - System parameters } \\
\text { [49] }\end{array}$ & ISO/TC204 & TS \\
\hline & $\begin{array}{l}\text { ISO/CD TS } 17264 \text { Road transport and traffic } \\
\text { telematics - Automatic vehicle and equipment } \\
\text { identification - Interfaces [50] }\end{array}$ & ISO/TC204 & $\begin{array}{l}\text { TS, } \\
\text { CD }\end{array}$ \\
\hline
\end{tabular}

\subsubsection{Transportation identification}

Transportation ID standards specify electronic ID cards, biometric data, and personal profile data exchange. 


\begin{tabular}{|c|c|c|c|}
\hline \multicolumn{2}{|r|}{ Transportation Identification } & SDO & Status \\
\hline \multirow{12}{*}{ ID card } & $\begin{array}{l}\text { ISO/IEC } 24727 \text { Identification cards - Integrated } \\
\text { circuit card programming interfaces - Part 1: } \\
\text { Architecture [104] }\end{array}$ & $\begin{array}{l}\text { ISO/IEC } \\
\text { JTC1/SC17 }\end{array}$ & IS \\
\hline & $\begin{array}{l}\text { ISO/IEC FCD } 24727 \text { Identification cards - } \\
\text { Integrated circuit card programming interfaces - } \\
\text { Part 2: Generic card interface [105] }\end{array}$ & $\begin{array}{l}\text { ISO/IEC } \\
\text { JTC1/SC17 }\end{array}$ & FCD \\
\hline & $\begin{array}{l}\text { ISO/IEC CD } 24727 \text { Identification cards - } \\
\text { Integrated circuit card programming interfaces - } \\
\text { Part 3: Application interface [106] }\end{array}$ & $\begin{array}{l}\text { ISO/IEC } \\
\text { JTC1/SC17 }\end{array}$ & CD \\
\hline & $\begin{array}{l}\text { ISO/IEC NP } 24727 \text { Identification Cards - } \\
\text { Programming Interfaces for Integrated Circuit } \\
\text { Cards - Part 4: API administration [107] }\end{array}$ & $\begin{array}{l}\text { ISO/IEC } \\
\text { JTC1/SC17 }\end{array}$ & NP \\
\hline & $\begin{array}{l}\text { ISO/IEC NP } 24727 \text { Identification Cards - } \\
\text { Programming Interfaces for Integrated Circuit } \\
\text { Cards - Part 5: Testing [108] }\end{array}$ & $\begin{array}{l}\text { ISO/IEC } \\
\text { JTC1/SC17 }\end{array}$ & NP \\
\hline & $\begin{array}{l}\text { ISO/IEC NP } 24749 \text { Identification cards - Secure } \\
\text { and interoperable IC card transaction device } \\
\text { [108] }\end{array}$ & $\begin{array}{l}\text { ISO/IEC } \\
\text { JTC1/SC17 }\end{array}$ & NP \\
\hline & $\begin{array}{l}\text { ISO/IEC } 14443 \text { Identification cards - Contactless } \\
\text { integrated circuit(s) cards - Proximity cards - Part } \\
\text { 1: Physical characteristics [22] }\end{array}$ & $\begin{array}{l}\text { ISO/IEC } \\
\text { JTC1/SC17 }\end{array}$ & IS \\
\hline & $\begin{array}{l}\text { ISO/IEC } 14443 \text { Identification cards - Contactless } \\
\text { integrated circuit(s) cards - Proximity cards - Part } \\
\text { 2: Radio frequency power and signal interface } \\
\text { [23] }\end{array}$ & $\begin{array}{l}\text { ISO/IEC } \\
\text { JTC1/SC17 }\end{array}$ & IS \\
\hline & $\begin{array}{l}\text { ISO/IEC } 14443 \text { Identification cards - Contactless } \\
\text { integrated circuit(s) cards - Proximity cards - Part } \\
\text { 3: Initialization and anticollision [25] }\end{array}$ & $\begin{array}{l}\text { ISO/IEC } \\
\text { JTC1/SC17 }\end{array}$ & IS \\
\hline & $\begin{array}{l}\text { ISO/IEC } 14443 \text { Identification cards - Contactless } \\
\text { integrated circuit(s) cards - Proximity cards - Part } \\
\text { 4: Transmission protocol [28] }\end{array}$ & $\begin{array}{l}\text { ISO/IEC } \\
\text { JTC1/SC17 }\end{array}$ & IS \\
\hline & $\begin{array}{l}\text { ISO/IEC 14443/Amd 1:2005 Bit rates of fc/64, } \\
\text { fc/32 and fc/16 [24] }\end{array}$ & $\begin{array}{l}\text { ISO/IEC } \\
\text { JTC1/SC17 }\end{array}$ & IS \\
\hline & $\begin{array}{l}\text { ISO/IEC 14443/Amd 3:2006 Handling of } \\
\text { reserved fields and values [27] }\end{array}$ & $\begin{array}{l}\text { ISO/IEC } \\
\text { JTC1/SC17 }\end{array}$ & IS \\
\hline Biometric data & $\begin{array}{l}\text { ISO/IEC } 19794 \text { Information technology - } \\
\text { Biometric data interchange formats - Part 1: } \\
\text { Framework [77] }\end{array}$ & $\begin{array}{l}\text { ISO/IEC } \\
\text { JTC1/SC37 }\end{array}$ & IS \\
\hline
\end{tabular}




\begin{tabular}{|c|c|c|c|}
\hline \multicolumn{2}{|r|}{ Transportation Identification } & \multirow{2}{*}{$\begin{array}{c}\text { SDO } \\
\text { ISO/IEC } \\
\text { JTC1/SC37 }\end{array}$} & \multirow{2}{*}{$\begin{array}{c}\text { Status } \\
\text { IS }\end{array}$} \\
\hline & $\begin{array}{l}\text { ISO/IEC } 19794 \text { Information technology - } \\
\text { Biometric data interchange formats - Part 2: } \\
\text { Finger minutiae data [79] }\end{array}$ & & \\
\hline & $\begin{array}{l}\text { ISO/IEC } 19794 \text { Information technology - } \\
\text { Biometric data interchange formats - Part 3: } \\
\text { Finger pattern spectral data [80] }\end{array}$ & $\begin{array}{l}\text { ISO/IEC } \\
\text { JTC1/SC37 }\end{array}$ & IS \\
\hline & $\begin{array}{l}\text { ISO/IEC } 19794 \text { Information technology - } \\
\text { Biometric data interchange formats - Part 4: } \\
\text { Finger image [81] }\end{array}$ & $\begin{array}{l}\text { ISO/IEC } \\
\text { JTC1/SC37 }\end{array}$ & IS \\
\hline & $\begin{array}{l}\text { ISO/IEC } 19794 \text { Information technology - } \\
\text { Biometric data interchange formats - Part 5: Face } \\
\text { image data [82] }\end{array}$ & $\begin{array}{l}\text { ISO/IEC } \\
\text { JTC1/SC37 }\end{array}$ & IS \\
\hline & $\begin{array}{l}\text { ISO/IEC 19794/FPDAmd } 1 \text { Conditions for taking } \\
\text { photographs for face image data [83] }\end{array}$ & $\begin{array}{l}\text { ISO/IEC } \\
\text { JTC1/SC37 }\end{array}$ & IS \\
\hline & $\begin{array}{l}\text { ISO/IEC } 19794 \text { Information technology -- } \\
\text { Biometric data interchange formats - Part 6: Iris } \\
\text { image data [84] }\end{array}$ & $\begin{array}{l}\text { ISO/IEC } \\
\text { JTC1/SC37 }\end{array}$ & IS \\
\hline & $\begin{array}{l}\text { ISO/IEC } 19794 \text { Information technology - } \\
\text { Biometric data interchange formats - Part 7: } \\
\text { Signature/sign time series data [85] }\end{array}$ & $\begin{array}{l}\text { ISO/IEC } \\
\text { JTC1/SC37 }\end{array}$ & IS \\
\hline & $\begin{array}{l}\text { ISO/IEC } 19794 \text { Information technology - } \\
\text { Biometric data interchange formats - Part 8: } \\
\text { Finger pattern skeletal data [86] }\end{array}$ & $\begin{array}{l}\text { ISO/IEC } \\
\text { JTC1/SC37 }\end{array}$ & IS \\
\hline \multirow{2}{*}{$\begin{array}{l}\text { Profile data } \\
\text { exchange }\end{array}$} & $\begin{array}{l}\text { ISO/IEC } 24713 \text { Information technology - } \\
\text { Biometric profiles for interoperability and data } \\
\text { interchange - Part 1: Biometric system reference } \\
\text { architecture [102] }\end{array}$ & $\begin{array}{l}\text { ISO/IEC } \\
\text { JTC1/SC37 }\end{array}$ & FCD \\
\hline & $\begin{array}{l}\text { ISO/IEC } 24713 \text { Biometric Profiles for } \\
\text { Interoperability and Data Interchange - Part 3: } \\
\text { Biometric Based Verification and Identification } \\
\text { of Seafarers [103] }\end{array}$ & $\begin{array}{l}\text { ISO/IEC } \\
\text { JTC1/SC37 }\end{array}$ & NP \\
\hline
\end{tabular}

\subsection{Port Access}

All the RFID, ID card, biometric data, and profile data exchange standards apply to port access security. Additionally, there is one more standard for port facility security assessment and planning. 


\begin{tabular}{|c|l|c|c|}
\hline \multicolumn{2}{|c|}{ Port Access } & SDO & Status \\
\hline \hline \multirow{2}{*}{$\begin{array}{c}\text { Port } \\
\text { facility }\end{array}$} & $\begin{array}{l}\text { ISO 20858 Ships and marine technology - Maritime port } \\
\text { facility security assessments and security plan } \\
\text { development [87] }\end{array}$ & $\begin{array}{c}\text { ISO/ } \\
\text { TC8 }\end{array}$ & PAS \\
\hline
\end{tabular}

\subsection{Communication in Supply Chain}

Communication in a supply chain involves medium and long range monitoring, tracking, electronic business transactions, and supply chain security.

\subsubsection{Medium and Long-Range Communication}

Currently, one locating system standard is available. A set of standards for air interface communication between devices is also available.

\begin{tabular}{|c|l|c|c|}
\hline \multicolumn{2}{|c|}{ Medium and Long-Range Communication } & \multicolumn{1}{c|}{ SDO } & Status \\
\hline \multirow{5}{*}{$\begin{array}{c}\text { Locating } \\
\text { systems }\end{array}$} & $\begin{array}{l}\text { ISO/IEC 24730 Information technology - Real- } \\
\text { time Locating Systems (RTLS) - Part 1: } \\
\text { Application program interface (API) }\end{array}$ & $\begin{array}{l}\text { ISO/IEC } \\
\text { JTC1/SC31 }\end{array}$ & IS \\
\cline { 2 - 4 } & $\begin{array}{l}\text { ISO/IEC 24730 Information technology - Real- } \\
\text { time Locating Systems (RTLS) - Part 2: 2.4 GHz } \\
\text { air Interface protocol }\end{array}$ & $\begin{array}{c}\text { ISO/IEC } \\
\text { JTC1/SC31 }\end{array}$ & FDIS \\
\cline { 2 - 4 } $\begin{array}{l}\text { ISO/IEC 24730 Information technology - Real- } \\
\text { tir interface } \\
\text { for } \\
\text { communication } \\
\text { Locating Systems (GLS) }\end{array}$ & $\begin{array}{l}\text { ISO/CD 21210 Communication air-interface } \\
\text { long and medium range (CALM) networking } \\
\text { protocols, Part 1: CALM Networking for } \\
\text { Internet Connectivity [88] }\end{array}$ & ISO/IEC & NP \\
\cline { 2 - 4 } & $\begin{array}{l}\text { ISO/CD 21210 CALM (Communication Air- } \\
\text { interface Long and Medium range) Networking } \\
\text { Protocols - Part 2: CALM Networking for Direct } \\
\text { Mode Connectivity [89] }\end{array}$ & IS \\
\cline { 2 - 4 } & $\begin{array}{l}\text { ISO/TC204 21212 Intelligent transport systems - } \\
\text { Communications, air-interface, long and medium } \\
\text { range (CALM) - 2G Cellular systems [90] }\end{array}$ & CD \\
\cline { 2 - 4 } & $\begin{array}{l}\text { ISO/DIS 21213 Intelligent transport systems - } \\
\text { Communications, air-interface, long and medium } \\
\text { range (CALM) - 3G Cellular systems [91] }\end{array}$ & ISO/TC204 & DIS \\
\hline
\end{tabular}




\begin{tabular}{|l|l|c|c|}
\hline \multicolumn{2}{|c|}{ Medium and Long-Range Communication } & SDO & Status \\
\hline \hline & $\begin{array}{l}\text { ISO 21216 CALM-MM: Medium and long } \\
\text { range, high speed, air interface parameters and } \\
\text { protocols for broadcast, point-point, vehicle- } \\
\text { vehicle, and vehicle-point communications in the } \\
\text { ITS Sector using millimeter wave microwave } \\
\text { communications, including specifications for } \\
\text { Master/Slave and Peer to Peer Communication } \\
\text { [92] }\end{array}$ & ISO/TC204 & WD \\
\cline { 2 - 4 } & $\begin{array}{l}\text { ISO/CD 21217 Communications, Air Interface, } \\
\text { Long and Medium Range (CALM) - } \\
\text { Architecture [93] }\end{array}$ & ISO/TC204 & CD \\
\cline { 2 - 4 } & $\begin{array}{l}\text { ISO/CD 21218 CALM Common Station } \\
\text { Manager (Lower Level SAPs) [94] }\end{array}$ & ISO/TC204 & CD \\
\hline
\end{tabular}

\subsubsection{Supply Chain Security}

Standards for business transaction in supply chains and supply security are available. Several organizations are developing open XML-based business transaction standards, such as, for example, OASIS (http://www.oasis-open.org), OAGIS (http://www.openapplications.org), and GS1 (http://www.gs1.org).

\begin{tabular}{|c|l|c|c|}
\hline \multicolumn{2}{|l|}{ Supply Chain Security } & SDO & Status \\
\hline \multirow{3}{*}{$\begin{array}{c}\text { Business } \\
\text { transactions }\end{array}$} & $\begin{array}{l}\text { ISO 9735 Electronic data interchange for } \\
\text { administration, commerce, and transport - } \\
\text { Application level syntax rules (Syntax } \\
\text { version number: 4, Syntax release number: } \\
\text { 1) - Part 1: Syntax rules common to all parts } \\
\text { [11] }\end{array}$ & ISO/TC154 & IS \\
\cline { 2 - 4 } & $\begin{array}{l}\text { ISO 9735 Electronic data interchange for } \\
\text { administration, commerce and transport } \\
\text { (EDIFACT) - Application level syntax rules } \\
\text { (Syntax version number: 4, Syntax release } \\
\text { number: 1) - Part 2: Syntax rules specific to } \\
\text { batch EDI [12] }\end{array}$ & ISO/TC154 & IS \\
\cline { 2 - 4 } & $\begin{array}{l}\text { ISO 9735 Electronic data interchange for } \\
\text { administration, commerce and transport } \\
\text { (EDIFACT) - Application level syntax rules } \\
\text { (Syntax version number: 4, Syntax release } \\
\text { number: 1) - Part 3: Syntax rules specific to } \\
\text { interactive EDI [13] }\end{array}$ & ISO/TC154 & IS \\
\cline { 2 - 4 } & & \\
\hline ISO 9735 Electronic data interehange for & ISO/TC154 & IS \\
\hline
\end{tabular}




\begin{tabular}{|c|c|c|}
\hline Supply Chain Security & \multirow[t]{2}{*}{ SDO } & \multirow[t]{2}{*}{ Status } \\
\hline $\begin{array}{l}\text { administration, commerce and transport } \\
\text { (EDIFACT) - Application level syntax rules } \\
\text { (Syntax version number: 4, Syntax release } \\
\text { number: 1) - Part 4: Syntax and service } \\
\text { report message for batch EDI (message type } \\
\text { - CONTRL) [14] }\end{array}$ & & \\
\hline $\begin{array}{l}\text { ISO } 9735 \text { Electronic data interchange for } \\
\text { administration, commerce and transport - } \\
\text { Application level syntax rules (Syntax } \\
\text { version number: 4, Syntax release number: } \\
\text { 1) - Part 5: Security rules for batch EDI } \\
\text { (authenticity, integrity and non-repudiation } \\
\text { of origin) [15] }\end{array}$ & ISO/TC154 & IS \\
\hline $\begin{array}{l}\text { ISO } 9735 \text { Electronic data interchange for } \\
\text { administration, commerce and transport - } \\
\text { Application level syntax rules (Syntax } \\
\text { version number: 4, Syntax release number: } \\
\text { 1) - Part 6: Secure authentication and } \\
\text { acknowledgement message (message type - } \\
\text { AUTACK) [16] }\end{array}$ & ISO/TC154 & IS \\
\hline $\begin{array}{l}\text { ISO } 9735 \text { Electronic data interchange for } \\
\text { administration, commerce and transport } \\
\text { (EDIFACT) - Application level syntax rules } \\
\text { (Syntax version number: 4, Syntax release } \\
\text { number: 1) - Part 7: Security rules for batch } \\
\text { EDI (confidentiality) [17] }\end{array}$ & ISO/TC154 & IS \\
\hline $\begin{array}{l}\text { ISO } 9735 \text { Electronic data interchange for } \\
\text { administration, commerce and transport } \\
\text { (EDIFACT) - Application level syntax rules } \\
\text { (Syntax version number: 4, Syntax release } \\
\text { number: 1) - Part 8: Associated data in EDI } \\
\text { [18] }\end{array}$ & ISO/TC154 & IS \\
\hline $\begin{array}{l}\text { ISO } 9735 \text { Electronic data interchange for } \\
\text { administration, commerce and transport } \\
\text { (EDIFACT) - Application level syntax rules } \\
\text { (Syntax version number: 4, Syntax release } \\
\text { number: 1) - Part 9: Security key and } \\
\text { certificate management message (message } \\
\text { type- KEYMAN) [19] }\end{array}$ & ISO/TC154 & IS \\
\hline $\begin{array}{l}\text { ISO } 9735 \text { Electronic data interchange for } \\
\text { administration, commerce and transport } \\
\text { (EDIFACT) - Application level syntax rules }\end{array}$ & ISO/TC154 & IS \\
\hline
\end{tabular}




\begin{tabular}{|c|l|c|c|}
\hline \multicolumn{1}{|l|}{ Supply Chain Security } & SDO & Status \\
\hline \multirow{6}{*}{} & $\begin{array}{l}\text { (Syntax version number: 4, Syntax release } \\
\text { number: 1) - Part 10: Syntax service } \\
\text { directories [20] }\end{array}$ & & \\
\hline \multirow{5}{*}{ Security } & $\begin{array}{l}\text { ISO 28000 Specification for Security } \\
\text { Management Systems for the Supply Chain } \\
\text { [118][119] }\end{array}$ & ISO/TC8 & DIS \\
\cline { 2 - 4 } & $\begin{array}{l}\text { ISO 28001 Ships and Marine Technology - } \\
\text { Best Practice For Implementing Supply } \\
\text { Chain Security - Assessments and Plans } \\
\text { [120] }\end{array}$ & ISO/TC8/SC111 & CD \\
\cline { 2 - 4 } $\begin{array}{l}\text { ISO 28003 Security Management Systems - } \\
\text { Requirements for Bodies Providing Audit } \\
\text { and Certification of Supply Chain Security } \\
\text { Management Systems [122] }\end{array}$ & ISO/TC8 & DIS \\
\cline { 2 - 4 } $\begin{array}{l}\text { ISO 28004 Security Management Systems } \\
\text { for the Supply Chain - Guidelines for the } \\
\text { Implementation of ISO/PAS 28000 [123] } \\
\text { [124] }\end{array}$ & ISO/TC8 & \\
\cline { 2 - 4 } $\begin{array}{l}\text { ISO/PAS 20858 Ships and Marine } \\
\text { Technology - Maritime Prot Facility } \\
\text { Security Assessments and Security Plan } \\
\text { Development [87] }\end{array}$ & ISO/TC8/SC11 & \\
\hline
\end{tabular}

\section{Conclusion}

Container security is a critical element of homeland security efforts to prevent the smuggling of weapons of mass destruction. Devices and information systems are used by container carriers, custodians, and Customs officials to detect broken seals and trace containers during shipping. Uninterrupted data transferring among these devices and systems are needed. Information and communication standards play a pivotal role in defining data models and communication protocols. Currently available standards and standard development bodies have been reviewed in this report. There are available standards for security in container seals, identification, transportation, remote communication, and supply chain. Some standards are in draft form and being developed.

Standards on some technical areas are still needed, such as sensor integration and the chain of custodianship. The integration of sensor and RFID technology must be in place to report tampering more effectively. Security information associated with container routing will ensure speedy pass-through inspection. Also, accessibility of security information to authorized personnel, such as carriers, port authorities, and Customs, must also be in place. Integration of security information is also needed. Lastly, and possibly more importantly, a workshop should be conducted to document requirements of new standards and to update some existing standards. The development of new standards and updating existing standards should be prioritized. After that, an information architecture 
for secure containers should be designed and agreed upon by all the parties who ensure the security.

\section{Acknowledgements}

The funding for this work was provided by the Department of Homeland Security. Authors gratefully acknowledge many suggestions and helpful comments from Steve Fick and Kang Lee of NIST.

\section{References}

[1] "Container Security Initiative 2006 - 2011 Strategic Plan", U.S. Customs and Border Protection Agency, Department of Homeland Security, http://www.cbp.gov/linkhandler/cgov/border_security/international_activities/csi/ csi_strategic_plan.ctt/csi_strategic_plan.pdf.

[2] Harmon, C., "An update on ISO activities regarding electronic container seals," Port Technology International, Issue 29, 14/1, 2006, pp. 1-3.

[3] Rand Corporation Europe Systems, “Securing the Supply Chain,” January 2005.

[4] International Organization for Standardization (ISO), Geneva, Switzerland, http://www.iso.org.

[5] International Electrotechnical Commission (IEC), Geneva, Switzerland, http://www.iec.ch.

[6] "Framework of Standards to Secure and Facilitate Global Trade," the World Customs Organization, June 2005.

[7] ISO 830 Freight containers - Vocabulary, International Standardization Organization, Geneva, Switzerland, 2004.

[8] ISO 1496 Series 1 freight containers - Specification and testing - Part 1: General cargo containers for general purposes, International Standardization Organization, Geneva, Switzerland, 2000.

[9] ISO 1496-1/Amd 5:2006 Door end security, International Standardization Organization, Geneva, Switzerland, 2006.

[10] ISO 6346 Freight containers - Coding, identification and marking, International Standardization Organization, Geneva, Switzerland, 1995.

[11] ISO 9735 Electronic data interchange for administration, commerce and transport (EDIFACT) - Application level syntax rules (Syntax version number: 4, Syntax release number: 1) - Part 1: Syntax rules common to all parts, International Standardization Organization, Geneva, Switzerland, 2002.

[12] ISO 9735 Electronic data interchange for administration, commerce and transport (EDIFACT) - Application level syntax rules (Syntax version number: 4, Syntax release number: 1) - Part 2: Syntax rules specific to batch EDI, International Standardization Organization, Geneva, Switzerland, 2002.

[13] ISO 9735-3:2002 Electronic data interchange for administration, commerce and transport (EDIFACT) - Application level syntax rules (Syntax version number: 4, Syntax release number: 1) - Part 3: Syntax rules specific to interactive EDI, International Standardization Organization, Geneva, Switzerland, 2002. 
[14] ISO 9735 Electronic data interchange for administration, commerce and transport (EDIFACT) - Application level syntax rules (Syntax version number: 4, Syntax release number: 1) - Part 4: Syntax and service report message for batch EDI (message type - CONTRL), International Standardization Organization, Geneva, Switzerland, 2002.

[15] ISO 9735 Electronic data interchange for administration, commerce and transport (EDIFACT) - Application level syntax rules (Syntax version number: 4, Syntax release number: 1) - Part 5: Security rules for batch EDI (authenticity, integrity and non-repudiation of origin), International Standardization Organization, Geneva, Switzerland, 2002.

[16] ISO 9735 Electronic data interchange for administration, commerce and transport (EDIFACT) - Application level syntax rules (Syntax version number: 4, Syntax release number: 1) - Part 6: Secure authentication and acknowledgement message (message type - AUTACK), International Standardization Organization, Geneva, Switzerland, 2002.

[17] ISO 9735 Electronic data interchange for administration, commerce and transport (EDIFACT) - Application level syntax rules (Syntax version number: 4, Syntax release number: 1) - Part 7: Security rules for batch EDI (confidentiality), International Standardization Organization, Geneva, Switzerland, 2002.

[18] ISO 9735 Electronic data interchange for administration, commerce and transport (EDIFACT) - Application level syntax rules (Syntax version number: 4, Syntax release number: 1) - Part 8: Associated data in EDI, International Standardization Organization, Geneva, Switzerland, 2002.

[19] ISO 9735 Electronic data interchange for administration, commerce and transport (EDIFACT) - Application level syntax rules (Syntax version number: 4, Syntax release number: 1) - Part 9: Security key and certificate management message (message type- KEYMAN), International Standardization Organization, Geneva, Switzerland, 2002.

[20] ISO 9735 Electronic data interchange for administration, commerce and transport (EDIFACT) - Application level syntax rules (Syntax version number: 4, Syntax release number: 1) - Part 10: Syntax service directories, International Standardization Organization, Geneva, Switzerland, 2002.

[21] ISO/CD 10374 Freight Container - RF automatic identification, International Standardization Organization, Geneva, Switzerland, 2006.

[22] ISO/IEC 14443 Identification cards - Contactless integrated circuit(s) cards Proximity cards - Part 1: Physical characteristics, International Standardization Organization, Geneva, Switzerland, 2003.

[23] ISO/IEC 14443 Identification cards - Contactless integrated circuit(s) cards Proximity cards - Part 2: Radio frequency power and signal interface, International Standardization Organization, Geneva, Switzerland, 2006.

[24] ISO/IEC 14443/Amd 1:2005 Bit rates of fc/64, fc/32 and fc/16, International Standardization Organization, Geneva, Switzerland, 2005.

[25] ISO/IEC 14443 Identification cards - Contactless integrated circuit(s) cards Proximity cards - Part 3: Initialization and anticollision, International Standardization Organization, Geneva, Switzerland, 2006. 
[26] ISO/IEC 14443/Amd 1:2005 Bit rates of fc/64, fc/32 and fc/16, International Standardization Organization, Geneva, Switzerland, 2005.

[27] ISO/IEC 14443/Amd 3:2006 Handling of reserved fields and values, International Standardization Organization, Geneva, Switzerland, 2006.

[28] ISO/IEC 14443 Identification cards - Contactless integrated circuit(s) cards Proximity cards - Part 4: Transmission protocol, International Standardization Organization, Geneva, Switzerland, 2006.

[29] ISO 14814 Road transport and traffic telematics - Automatic vehicle and equipment identification - Reference architecture and terminology, International Standardization Organization, Geneva, Switzerland, 2006.

[30] ISO 14815 Road transport and traffic telematics - Automatic vehicle and equipment identification - System specifications, International Standardization Organization, Geneva, Switzerland, 2005.

[31] ISO/TS 14816 Road transport and traffic telematics - Automatic vehicle and equipment identification - Numbering and data structure, International Standardization Organization, Geneva, Switzerland, 2005.

[32] ISO/TR 15070 Series 1 freight containers - Rationale for structural test criteria, International Standardization Organization, Geneva, Switzerland, 2005.

[33] ISO/TR 15070/Amd 1:2005 Guidance on structural integrity, International Standardization Organization, Geneva, Switzerland, 2005.

[34] ISO/TR 15070/1996/CD Amd 2 Design Consideration, 2007.

[35] ISO/IEC 15418 Information technology - EAN/UCC Application Identifiers and Fact Data Identifiers and Maintenance, International Standardization Organization, Geneva, Switzerland, 2005.

[36] ISO/IEC 15424 Information technology - Automatic identification and data capture techniques - Data Carrier Identifiers, International Standardization Organization, Geneva, Switzerland, 2005.

[37] ISO/IEC 15434 Information technology - Automatic identification and data capture techniques - Syntax for high-capacity ADC media, International Standardization Organization, Geneva, Switzerland, 2006.

[38] ISO/IEC 15459 Information technology - Unique identifiers - Part 1: Unique identifiers for transport units, International Standardization Organization, Geneva, Switzerland, 2006.

[39] ISO/IEC 15459 Information technology - Unique identifiers - Part 2: Registration procedures, International Standardization Organization, Geneva, Switzerland, 2006.

[40] ISO/IEC 15459 Information technology - Unique identifiers - Part 3: Common rules for unique identifiers, International Standardization Organization, Geneva, Switzerland, 2006.

[41] ISO/IEC 15459 Information technology - Unique identifiers - Part 4: Unique identifiers for supply chain management, International Standardization Organization, Geneva, Switzerland, 2006.

[42] ISO/IEC FCD 15459 Information technology - Unique identification of transport units - Part 5: Unique identification of returnable transport items (RTIs) (under development), International Standardization Organization, Geneva, Switzerland, 2006. 
[43] ISO/IEC FCD 15459 Information technology - Unique identification of transport units - Part 6: Unique identification for product groupings in material lifecycle management (under development), International Standardization Organization, Geneva, Switzerland, 2006.

[44] ISO/IEC 15961, Information technology - Radio frequency identification (RFID) for item management - Data protocol: application interface, International Standardization Organization, Geneva, Switzerland, 2004.

[45] ISO/IEC 15962, Information technology - Radio frequency identification (RFID) for item management - Data protocol: data encoding rules and logical memory functions, International Standardization Organization, Geneva, Switzerland, 2004.

[46] ISO/IEC 15963, Information technology - Radio frequency identification for item management - Unique identification for RF tags, International Standardization Organization, Geneva, Switzerland, 2004.

[47] ISO/TS 17261, Intelligent transport systems - Automatic vehicle and equipment identification - Intermodal goods transport architecture and terminology, International Standardization Organization, Geneva, Switzerland, May 2005.

[48] ISO/TS 17262, Intelligent transport systems - Automatic vehicle and equipment identification - Intermodal goods transport - Numbering and data structures, International Standardization Organization, Geneva, Switzerland, June 2003.

[49] ISO/TS 17263, Intelligent transport systems - Automatic vehicle and equipment identification - Intermodal goods transport - System parameters, International Standardization Organization, Geneva, Switzerland, June 2003.

[50] ISO/CD TS 17264, Road transport and traffic telematics - Automatic vehicle and equipment identification - Interfaces, International Standardization Organization, Geneva, Switzerland, 2006.

[51] ISO/DIS 17363 Supply chain application of RFID - Freight containers, International Standardization Organization, Geneva, Switzerland, 2006.

[52] ISO/DIS 17364 Supply chain applications of RFID - Returnable transport items (RTIs), International Standardization Organization, Geneva, Switzerland, 2006.

[53] ISO/DIS 17365 Supply chain applications of RFID - Transport units, International Standardization Organization, Geneva, Switzerland, 2006.

[54] ISO/DIS 17366.2 Supply chain applications of RFID - Product packaging, International Standardization Organization, Geneva, Switzerland, 2006.

[55] ISO/DIS 17367.2 Supply chain applications of RFID - Product tagging , 2006.

[56] ISO/FDIS 17687 Transport Information Systems - General Fleet Management and Commercial Freight Operations - Data Dictionary and Message Sets for Electronic Identification and Monitoring of Hazardous Materials/Dangerous Goods Transportation, International Standardization Organization, Geneva, Switzerland, 2006.

[57] ISO/PAS 17712 Freight containers - Mechanical seals, International Standardization Organization, Geneva, Switzerland, 2006.

[58] ISO/IEC 18000-1 Information Technology - Radio frequency identification for item management - Part 1: Reference architecture and definition of parameters to be standardize, International Standardization Organization, Geneva, Switzerland, 2004. 
[59] ISO/IEC 18000 Information Technology - Radio frequency identification for item management - Part 2: Parameters for air interface communication below $135 \mathrm{kHz}$, International Standardization Organization, Geneva, Switzerland, 2004.

[60] ISO/IEC 18000 Information Technology - Radio frequency identification for item management - Part 3: Parameters for air interface communication below 13,56 MHz, International Standardization Organization, Geneva, Switzerland, 2004.

[61] ISO/IEC 18000 Information Technology - Radio frequency identification for item management - Part 4: Parameters for air interface communication below 2,45 GHz, International Standardization Organization, Geneva, Switzerland, 2004.

[62] ISO/IEC 18000 Information Technology - Radio frequency identification for item management - Part 6: Parameters for air interface communication at $860 \mathrm{MHz}$ to $960 \mathrm{MHz}$, International Standardization Organization, Geneva, Switzerland, 2004.

[63] ISO/IEC 18000-6:2004/Amd 1 Extension with Type C and update of Types A and B, International Standardization Organization, Geneva, Switzerland, 2006.

[64] ISO/IEC FDIS 18000 Information technology - Radio frequency identification for item management - Part 7: Parameters for active air interface communications at $433 \mathrm{MHz}$, International Standardization Organization, Geneva, Switzerland, 2006.

[65] ISO/IEC 18046 Information technology - Automatic identification and data capture techniques - Radio frequency identification device performance test methods, International Standardization Organization, Geneva, Switzerland, 2006.

[66] ISO/IEC TR 18047 Information technology - Radio frequency identification device conformance test methods - Part 2: Test methods for air interface communications below $135 \mathrm{kHz}$, International Standardization Organization, Geneva, Switzerland, 2006.

[67] ISO/IEC TR 18047 Information technology - Radio frequency identification device conformance test methods - Part 3: Test methods for air interface communications at 13,56 MHz, International Standardization Organization, Geneva, Switzerland, 2004. (

[68] ISO/IEC TR 18047 Information technology - Radio frequency identification device conformance test methods - Part 4: Test methods for air interface communications at 2,45 GHz, International Standardization Organization, Geneva, Switzerland, 2004.

[69] ISO/IEC TR 18047 Information technology - Radio frequency identification device conformance test methods - Part 6: Test methods for air interface communications at $860 \mathrm{MHz}$ to $960 \mathrm{MHz}$, International Standardization Organization, Geneva, Switzerland, 2006.

[70] ISO/IEC TR 18047 Information technology - Radio frequency identification device conformance test methods - Part 7: Test methods for active air interface communications at $433 \mathrm{MHz}$, International Standardization Organization, Geneva, Switzerland, 2006.

[71] ISO/FDIS 18185 Freight containers - Electronic seals - Part 1: Communication protocol, International Standardization Organization, Geneva, Switzerland, 2006.

[72] ISO/FDIS 18185 Freight containers - Electronic seals - Part 2: Application requirements, International Standardization Organization, Geneva, Switzerland, 2007. 
[73] ISO 18185 Freight containers - Electronic seals - Part 3: Environment characteristics, International Standardization Organization, Geneva, Switzerland, 2006.

[74] ISO/FDIS 18185 Freight containers - Electronic seals - Part 4: Data protection, International Standardization Organization, Geneva, Switzerland, 2007.

[75] ISO/FDIS 18185 Freight containers - Electronic seals - Part 5: Sensor interface, International Standardization Organization, Geneva, Switzerland, 2007.

[76] ISO/CD 18185 Freight containers - Electronic seals - Part 6 Message sets for transfer between seal reader and host computer, International Standardization Organization, Geneva, Switzerland, 2006.

[77] ISO/DIS 18185 Freight containers - Electronic seals - Part 7 Physical layer, International Standardization Organization, Geneva, Switzerland, 2006.

[78] ISO/IEC 19794 Information technology - Biometric data interchange formats Part 1: Framework, International Standardization Organization, Geneva, Switzerland, 2006.

[79] ISO/IEC 19794 Information technology - Biometric data interchange formats Part 2: Finger minutiae data, International Standardization Organization, Geneva, Switzerland, 2005.

[80] ISO/IEC 19794 Information technology - Biometric data interchange formats Part 3: Finger pattern spectral data, International Standardization Organization, Geneva, Switzerland, 2006.

[81] ISO/IEC 19794 Information technology - Biometric data interchange formats Part 4: Finger image, International Standardization Organization, Geneva, Switzerland, 2005.

[82] ISO/IEC 19794 Information technology - Biometric data interchange formats Part 5: Face image data, International Standardization Organization, Geneva, Switzerland, 2005.

[83] ISO/IEC 19794/FPDAmd 1 Conditions for taking photographs for face image data, International Standardization Organization, Geneva, Switzerland, 2006.

[84] ISO/IEC 19794 Information technology -- Biometric data interchange formats Part 6: Iris image data, International Standardization Organization, Geneva, Switzerland, 2005.

[85] ISO/IEC 19794-7.2 Information technology - Biometric data interchange formats - Part 7: Signature/sign time series data, International Standardization Organization, Geneva, Switzerland, 2007.

[86] ISO/IEC 19794-8 Information technology - Biometric data interchange formats Part 8: Finger pattern skeletal data, International Standardization Organization, Geneva, Switzerland, 2006.

[87] ISO/PAS 20858 Ships and marine technology - Maritime port facility security assessments and security plan development, International Standardization Organization, Geneva, Switzerland, 2004.

[88] ISO/CD 21210 CALM (Communication Air-interface Long and Medium range) Networking Protocols - Part 1: CALM Networking for Internet Connectivity, International Standardization Organization, Geneva, Switzerland, 2006. 
[89] ISO/CD 21210 CALM (Communication Air-interface Long and Medium range) Networking Protocols - Part 2: CALM Networking for Direct Mode Connectivity, International Standardization Organization, Geneva, Switzerland, 2006.

[90] ISO/DIS 21212 Intelligent transport systems - Communications, air-interface, long and medium range (CALM) - 2G Cellular systems, International Standardization Organization, Geneva, Switzerland, 2006.

[91] ISO/DIS 21213 Intelligent transport systems - Communications, air-interface, long and medium range (CALM) - 3G Cellular systems, International Standardization Organization, Geneva, Switzerland, 2006.

[92] ISO/WD 21216 CALM-MM: Medium and long range, high speed, air interface parameters and protocols for broadcast, point-point, vehicle-vehicle, and vehiclepoint communications in the ITS Sector using MILLIMETRE WAVE MOCROWAVE COMMUNICATIONS, including specifications for Master/Slave and Peer to Peer Communications, International Standardization Organization, Geneva, Switzerland, 2005

[93] ISO/CD 21217 Communications, Air Interface, Long and Medium Range (CALM) - Architecture, International Standardization Organization, Geneva, Switzerland, 2005.

[94] ISO/CD 21218 CALM Common Station Manager (Lower Level SAPs), International Standardization Organization, Geneva, Switzerland, 2006. (ISO TC204)

[95] ISO/FDIS 24531 Intelligent transport systems - System architecture, taxonomy and terminology - Using XML in ITS standards, data registries and data dictionaries, International Standardization Organization, Geneva, Switzerland, 2006.

[96] ISO/CD 24533 Transport information and control systems - Data dictionary and message set to facilitate the movement of freight and its intermodal transfer Road transport information exchanges, International Standardization Organization, Geneva, Switzerland, 2005.

[97] ISO/CD TS 24534 Automatic vehicle and equipment identification - Electronic Registration Identification (ERI) for vehicles - Part 1: Architecture, International Standardization Organization, Geneva, Switzerland, 2007.

[98] ISO/CD TS 24534 Automatic vehicle and equipment identification - Electronic Registration Identification (ERI) for vehicles - Part 2: Operational requirements, International Standardization Organization, Geneva, Switzerland, 2007.

[99] ISO/CD TS 24534 Automatic vehicle and equipment identification - Electronic Registration Identification (ERI) for vehicles - Part 3: Vehicle data, International Standardization Organization, Geneva, Switzerland, 2007.

[100] ISO/CD TS 24534 Automatic vehicle and equipment identification - Electronic Registration Identification (ERI) for vehicles - Part 4: Secure communications using asymmetrical techniques, International Standardization Organization, Geneva, Switzerland, 2007.

[101] ISO/DIS 24535 Intelligent transport systems - Automatic Vehicle Identification Basic electronic registration identification (Basic ERI), International Standardization Organization, Geneva, Switzerland, 2006. 
[102] ISO/IEC FCD 24713-1.2 Information technology - Biometric profiles for interoperability and data interchange - Part 1: Biometric system reference architecture, International Standardization Organization, Geneva, Switzerland, 2006.

[103] ISO/IEC NP 24713 Biometric Profiles for Interoperability and Data Interchange Part 3: Biometric Based Verification and Identification of Seafarers, International Standardization Organization, Geneva, Switzerland, 2004.

[104] ISO/IEC 24727 Identification cards - Integrated circuit card programming interfaces - Part 1: Architecture, International Standardization Organization, Geneva, Switzerland, 2007.

[105] ISO/IEC FCD 24727 Identification cards - Integrated circuit card programming interfaces - Part 2: Generic card interface, International Standardization Organization, Geneva, Switzerland, 2005.

[106] ISO/IEC CD 24727 Identification cards - Integrated circuit card programming interfaces - Part 3: Application interface, International Standardization Organization, Geneva, Switzerland, 2005.

[107] ISO/IEC NP 24727 Identification Cards - Programming Interfaces for Integrated Circuit Cards - Part 4: API administration, International Standardization Organization, Geneva, Switzerland, 2005.

[108] ISO/IEC NP 24727 Identification Cards - Programming Interfaces for Integrated Circuit Cards - Part 5: Testing, International Standardization Organization, Geneva, Switzerland, 2005.

[109] ISO/IEC CD TR 24729 Information technology - Radio frequency identification for item management - Implementation guidelines - Part 1: RFID-enabled labels, International Standardization Organization, Geneva, Switzerland, 2006.

[110] ISO/IEC CD TR 24729 Information technology - Radio frequency identification for item management - Implementation guidelines - Part 2: Recyclability of RF tags, International Standardization Organization, Geneva, Switzerland, 2006.

[111] ISO/IEC NP TR 24729 Information technology - Radio frequency identification for item management - Implementation guidelines - Part 3: RFID interrogator/antenna installation, International Standardization Organization, Geneva, Switzerland, 2005.

[112] ISO/IEC 24730 Information technology - Real-time Locating Systems (RTLS) Part 1: Application program interface (API), International Standardization Organization, Geneva, Switzerland, 2006.

[113] ISO/IEC FDIS 24730 Information technology - Real-time Locating Systems (RTLS) - Part 2: $2.4 \mathrm{GHz}$ air Interface protocol, International Standardization Organization, Geneva, Switzerland, 2006.

[114] ISO/IEC NP 24730 Information technology - Real-time Locating Systems (RTLS) - Part 4: Global Locating Systems (GLS), International Standardization Organization, Geneva, Switzerland, 2005.

[115] ISO/IEC NP 24749 Identification cards - Secure and interoperable IC card transaction device, International Standardization Organization, Geneva, Switzerland, 2004.

[116] ISO/IEC NP 24753 Automatic Identification and Data Capture Techniques Radio Frequency Identification (RFID) for Item Management - Air Interface for 
Battery Assist and Sensor Function, International Standardization Organization, Geneva, Switzerland, 2005.

[117] ISO/IEC NP 24791 Information technology - Automatic Identification and Data Capture Techniques - Radio-Frequency Identification (RFID) for Item Management - System Management Protocol, International Standardization Organization, Geneva, Switzerland, 2005.

[118] ISO/DIS 28000 Specification for security management systems for the supply chain International Standardization Organization, Geneva, Switzerland, 2006.

[119] ISO/PAS 28000 Specification for security management systems for the supply chain, International Standardization Organization, Geneva, Switzerland, 2005.

[120] ISO/CD 28001 Security management systems for the supply chain - Best practices for implementing supply chain security, assessments and plans Requirements and guidance, International Standardization Organization, Geneva, Switzerland, 2007.

[121] ISO/PAS 28001 Security management systems for the supply chain - Best practices for implementing supply chain security - Assessments and plans, International Standardization Organization, Geneva, Switzerland, 2006.

[122] ISO/DIS 28003 Security management systems for the supply chain Requirements for bodies providing audit and certification of supply chain security management systems, International Standardization Organization, Geneva, Switzerland, 2006.

[123] ISO/DIS 28004 Security management systems for the supply chain - Guidelines for the implementation of ISO/PAS 28000, International Standardization Organization, Geneva, Switzerland, 2006.

[124] ISO/PAS 28004 Security management systems for the supply chain - Guidelines for the implementation of ISO/PAS 28000, International Standardization Organization, Geneva, Switzerland, 2006. 